\title{
Functional relevance of IL-10 promoter polymorphisms for sepsis development
}

\author{
Spaska A Stanilova* \\ See related research by Zeng et al., http://ccforum.com/content/13/6/R188
}

\begin{abstract}
The induced production of proinflammatory and anti-inflammatory cytokines is considered important for the development of sepsis and its sequelae. Polymorphisms in the IL-10 gene promoter could influence its expression and sepsis susceptibility. Results obtained by Dr Ling and colleagues demonstrated that the $-1082 \mathrm{~A}$ allele was significantly associated with lower lipopolysaccharide-induced IL-10 production in an allele-dose-dependent fashion. They also showed that this polymorphism was significantly associated with sepsis development after major trauma. These and other research data clearly demonstrated that the $-1082 \mathrm{~A} / \mathrm{G}$ polymorphism in the IL-10 gene promoter has an important impact on susceptibility of sepsis and sepsis outcome.
\end{abstract}

Dr Ling and colleagues performed a large prospective study, including 308 patients with major trauma, and determined that the genotype distribution of the IL-10 polymorphisms was associated with the sepsis morbidity rate and multiple organ dysfunction syndrome (MODS) scores [1]. The authors also investigated the association of polymorphisms with lipopolysaccharide-induced IL10 production.

Cytokine production varies between individuals, due to genetic background and certain allelic variants of cytokine genes - in particular, SNPs are associated with higher or lower cytokine production. The IL-10 5'-flanking region, which controls transcription, is polymorphic, with two microsatellites and three SNPs $(-1082,-819$, and -592) [2]. Polymorphisms in the regulatory region of the IL-10 gene may influence its expression, and hence could influence the susceptibility of sepsis [3].

*Correspondence: stanilova@mf.uni-sz.bg

Department of Molecular Biology, Immunology \& Medical Genetics, Faculty of Medicine, Trakia University, Aremiska 11 str., Stara Zagora, Bulgaria
The authors investigated the hypothesis that SNPs located in the cis-regulatory region of IL-10 promoter might represent a risk factor or a protective factor for developing sepsis and might influence the MODS score after major trauma. The results obtained by Dr Ling and colleagues demonstrated that both the $-1082 \mathrm{~A}$ and -592A alleles were significantly associated with lower lipopolysaccharide-induced IL-10 production in an allele-dose-dependent fashion. In contrast, there was no significant difference for association between the -819 polymorphism and induced cytokine production. These results are in agreement with the data from Turner and colleagues, who found that the IL-10 $-1082 \mathrm{~A}$ allele is associated with lower in vitro IL-10 production by concanavalin-A-stimulated peripheral blood mononuclear cells from normal subjects [2]. Moreover, Stanilova and colleagues reported that carriage of at least one copy of the IL-10 -1082A allele in sepsis patients as well as in healthy controls resulted in a statistically significant decrease of IL-10 production from stimulated peripheral blood mononuclear cells, regardless of the stimuli used [4].

Altered host defense mechanisms are considered important for the development of sepsis and septic shock. Proinflammatory and anti-inflammatory responses contribute to the development and outcome of severe sepsis [5]. Cytokines play a pivotal role in the regulation of the type and magnitude of the immune response, and the polymorphic nature of the cytokine genes may confer flexibility on the immune response. The gene encoding IL-10 cytokine involved in the modulation of inflammatory responses as a main anti-inflammatory agent is therefore a candidate gene for determination of the human genetic background, which is responsible for interindividual differences in susceptibility to sepsis development. Previous reports have indicated that increased IL-10 production might be associated with the susceptibility and outcome of the sepsis [6-8]. The polymorphism at position -1082 in the promoter region of the IL-10 gene has been studied by Shu and colleagues, who reported an association of this polymorphism with 
susceptibility to severe sepsis - in contrast to the other two linked IL-10 polymorphisms (-592 and -819) in the same gene region [9]. Stanilova and colleagues showed that the A allele of the -1082 polymorphism in the IL-10 gene promoter is associated with sepsis susceptibility, whereas the $G$ allele is associated with higher stimulated IL-10 production and increased mortality in severe sepsis [4].

Ling and colleagues also showed that, unlike the -1082 polymorphism, the -819 and -592 polymorphisms were not significantly associated with the sepsis morbidity rate and MODS scores. These collective data clearly demonstrated that the $-1082 \mathrm{~A} / \mathrm{G}$ polymorphism in the IL-10 gene has an important impact on susceptibility of sepsis and sepsis outcome. This effect is due to the antiinflammatory and immunoregulatory properties of IL-10. The excess of IL-10 production in trauma patients or other critically ill patients determined by the $-1082 \mathrm{G}$ allele could be responsible for inducing immunosuppression and subsequently developing bacterial sepsis and MODS. The concentration of IL-10 in the blood - in most respects, lipopolysaccharide-induced IL-10 - is indicative for the magnitude of the inflammatory stress during sepsis and has been shown to correlate with both the severity and outcome of sepsis.

Whether cytokine determination and genotyping in critically ill patients could optimize treatment and sepsis outcome should be determined by further randomized studies.

\section{Abbreviations}

$\mathrm{IL}=$ interleukin; MODS = multiple organ dysfunction syndrome; SNP = single nucleotide polymorphism.

\section{Competing interests}

The author declares that they have no competing interests.

Published: 15 February 2010

\section{References}

1. Zeng L, Gu W, Chen K, Jiang D, Zhang L, Du D, Hu P, Liu Q, Huang S, Jiang J: Clinical relevance of the interleukin 10 promoter polymorphisms in Chinese Han patients with major trauma: genetic association studies. Crit Care 2009, 13:R188

2. Turner DM, Williams DM, Sankaran D, Lazarus M, Sinnott PJ, Hutchinson IV: An investigation of polymorphism in the interleukin-10 gene promoter. Eur J Immunogenet 1997, 24:1-8.

3. Stuber F: Effect of genomic polymorphisms on the course of sepsis: is there a concept for gene therapy? J Am Soc Nephrol 2001, 12:S60-S64.

4. Stanilova S, Miteva L, Karakolev Zh, Stefanov Ch: Interleukin-10-1082 promoter polymorphism in association with cytokine production and sepsis susceptibility. Intensive Care Med 2006, 32:260-266.

5. Gogos C, Drosou E, Bassaris H, Skoutelis A: Pro versus anti-inflammatory cytokine profile in patiens with severe sepsis: a marker for prognosis and future therapeutic options. J Infect Dis 2000, 181:176-180.

6. Latifi SQ, O'Riordan MA, Levine AD: Interleukin-10 controls the onset of irreversible septic shock. Infect Immun 2002, 70:4441-4446.

7. Stanilova SA, Karakolev ZT, Dimov GS, Dobreva ZG, Miteva LD, Slavov ES, Stefanov CS, Stanilov NS: High interleukin 12 and low interleukin 10 production after in vitro stimulation detected in sepsis survivors. Intensive Care Med 2005, 31:401-407.

8. Bozza F, Salluh J, Japiassu A, Soares M, Assis E, Gomes R, Bozza M, Castro-FariaNeto H, Bozza P: Cytokine profiles as markers of disease severity in sepsis: a multiplex analysis. Crit Care 2007, 11:R49.

9. Shu Q, Fang X, Chen Q, Stuber F: IL-10 polymorphism is associated with increased incidence of severe sepsis. Chin Med J (Engl) 2003, 116:1756-1759.

doi:10.1186/cc8839

Cite this article as: Stanilova SA: Functional relevance of IL-10 promoter polymorphisms for sepsis development. Critical Care 2010, 14:119. 